OPEN ACCESS

Edited by: Husain Yar Khan, Wayne State University, United States

Reviewed by: Zeeshan Ahmad, Wayne State University, United States Mitsuru Otsubo, Asahikawa Medical University, Japan

*Correspondence: Shibo Tang tangshibo@vip.163.com Jacey Hongjie Ma ma.hongjie12@gmail.com

${ }^{\dagger}$ These authors have contributed equally to this work and share first authorship

Specialty section: This article was submitted to Pharmacology of Anti-Cancer Drugs,

a section of the journal

Frontiers in Oncology

Received: 10 September 2021 Accepted: 22 November 2021 Published: 13 December 2021

Citation:

Ye S, Fang $Q$, Yao J, Xing J, Tang S and Ma JH (2021) Intravitreal Ranibizumab Had Limited Effect on Cystoid Macular Edema Due to Albumin-Bound Paclitaxel: A Case Report and Literature Review.

Front. Oncol. 11:773540. doi: 10.3389/fonc.2021.773540

\section{Intravitreal Ranibizumab Had Limited Effect on Cystoid Macular Edema Due to Albumin-Bound Paclitaxel: A Case Report and Literature Review}

\author{
Suna $\mathrm{Ye}^{1,2 \dagger}$, Qiqi Fang ${ }^{3 \dagger}$, Jinyu $\mathrm{Yao}^{3}$, Jianqiang Xing ${ }^{3}$, Shibo Tang ${ }^{1,2^{*}}$ \\ and Jacey Hongjie $\mathrm{Ma}^{1,2 *}$ \\ ${ }^{1}$ AlER Eye Hospital, Jinan University, Guangzhou, China, ${ }^{2}$ AlER Eye Institute, Changsha, China, ${ }^{3}$ Retina Department, Hainan \\ AIER Eye Hospital, Haikou, China
}

Angiographically silent cystoid macular edema $(\mathrm{CME})$ is a rare complication from nabpaclitaxel. Here we report a 45-year-old woman with breast cancer who developed CME after several months of treatment with albumin-bound paclitaxel (nab-paclitaxel). Her visual acuity did not improve significantly with the cessation of nab-paclitaxel and intravitreal ranibizumab treatment. Then, brinzolamide eye drops were prescribed. One month later, her vision improved, with the macular edema significantly subsided. Finally, we reviewed other cases of CME induced by nab-paclitaxel that have been reported in the literature and discussed the underlying pathogenesis of nab-paclitaxel-induced CME.

Keywords: albumin-bound paclitaxel, Nab-paclitaxel, Abraxane, cystoid macular edema, ranibizumab, carbonic anhydrase inhibitor

\section{INTRODUCTION}

Paclitaxel is a mitogenic inhibiting anti-microtubule agent used as first- or second-line treatment in various cancers, alone or in combination with other drugs. The most common indications of paclitaxel include breast cancer, ovarian cancer, and non-small cell lung cancer (1). Albumin-bound paclitaxel (nab-paclitaxel, Abraxane) is an albumin-stabilized paclitaxel nanoparticle formulation with potent antitumor effects than paclitaxel in metastatic breast cancer that has failed combination chemotherapy or recurred within 6 months of adjuvant chemotherapy (2). Cystoid macular edema $(\mathrm{CME})$ is a rare complication caused by nab-paclitaxel $(3,4)$. Here we report the first case of nabpaclitaxel-induced CME treated with intravitreal ranibizumab (IVR) and review the literature about nab-paclitaxel-related CME.

\section{CASE REPORT}

A 45-year-old female patient diagnosed with right breast cancer underwent a right lumpectomy in March 2016. The pathological examination revealed grade II non-specific invasive ductal carcinoma. The immunohistochemical staining results were as follows: ER (+), PR (-), and HER2(-). The patient received postoperative treatment with traditional Chinese medicine and 
tamoxifen. In January 2018, the cancer progressed and metastasized to the pectoralis major muscle, lymph nodes, and scapula. From January 2018 to July 2019, she received a chemotherapy regimen comprised of docetaxel, epirubicin, and cyclophosphamide. In July 2019, she started a T regimen nabpaclitaxel (200 mg weekly), which was lowered to 11 consecutive single doses of $170 \mathrm{mg}$ weekly due to adverse drug reactions. From November 12, 2019 to December 25, 2020, the chemotherapeutic regimen of the patient was changed to nabpaclitaxel (once at $400 \mathrm{mg}$ and then thrice at $340 \mathrm{mg}$, once every 3 weeks). It was then switched to $360 \mathrm{mg}$ once every 3 weeks for a total of 16 doses. On January 16, 2021, she received the last T regimen of nab-paclitaxel (400 $\mathrm{mg}$, cumulative dose: 9,650 mg).

In January 2021, the patient complained of gradually decreasing vision in both eyes over 4 weeks. Then, she received treatment and follow-up by ophthalmologists, and the time course is shown in Figure 1. At the initial ophthalmologic examination, the best-corrected visual acuity (BCVA) was 20/60 on the right and $20 / 100$ on the left, and color vision was intact. The anterior segment examination revealed diffuse corneal epithelial defects. The dilated fundus examination revealed macular edema in both eyes (Figures 2A, B). The fluorescein angiograms did not reveal leakage from the parafoveal capillaries in early or late stages (Figures 2C-F). The optical coherence tomography (OCT) and optical coherence tomography angiography (OCTA) scans on both eyes revealed CME with a thickened central retina (Figures 2G, H, 3A). Neither CME-related systemic nor intraocular disorder, such as diabetes, uveitis, or retinal vein occlusion, was detected after a detailed physical examination and fluorescent angiography. Moreover, she had not taken any other CME-related medications. Then, the diagnosis of taxane-related CME (TCME) was established.

Nab-paclitaxel was ceased after the CME was revealed, and 1 $\mathrm{mg} / 0.1 \mathrm{ml}$ of ranibizumab (Lucentis, $10 \mathrm{mg} / \mathrm{ml}$ ) was injected intravitreally in both eyes. However, at 1 month after applying
IVR, neither the BCVA of the patient nor the central retinal thinness detected by OCT was changed (Figures 1, 3A-a, f, 3B-g, 1). However, the area of macular edema was reduced (Figures 3A-b-e, B-h-k). Since the BCVA was not increased, the patient refused IVR treatment. Then, brinzolamide eye drops (Azopt, 10mg/ml, twice daily) were applied to both eyes. One month later, the BCVA had improved to 20/30 in both eyes, and OCT and OCTA revealed that the macular edema almost subsided in both central macular B-scan (Figure 3C-m, r) and en face OCT and OCTA (Figure 3C-n-q).

Written informed consent was obtained from the patient for the publication of any potentially identifiable images or data included in this article.

\section{LITERATURE REVIEW}

We performed a literature search in PubMed and Web of Science before July 30, 2021 for the following terms: "cystoid macular edema" AND ("nab-paclitaxel" or "albumin-bound paclitaxel" or "Abraxane"). A total of 22 nab-paclitaxel-related CME cases were identified in the literature review. The images, treatments, and recovery time of these cases and of our case are presented in Table $\mathbf{1 .}$

In these cases, the male to female ratio was 1:2.8, the mean age was 60 years (32-73 years), and almost all had bilateral eyes involvement except one. Fourteen cases were diagnosed with breast cancer $(7-17,24)$, six with pancreatic cancer (19-24), and the others with melanoma (5), cancer of the hypopharynx (18), and lung cancer (6), respectively. The treatment regimens were heterogeneous, with most patients discontinuing nab-paclitaxel with or without eye treatments, including topical nonsteroidal anti-inflammatory drugs (NSAIDs), topical corticosteroids, topical dorzolamide (DRZ), intravitreal bevacizumab (IVB), dexamethasone. Our case was the first patient treated with ranibizumab. We analyzed the recovery time course of all the cases, which are listed in Table 1. The recovery period in these

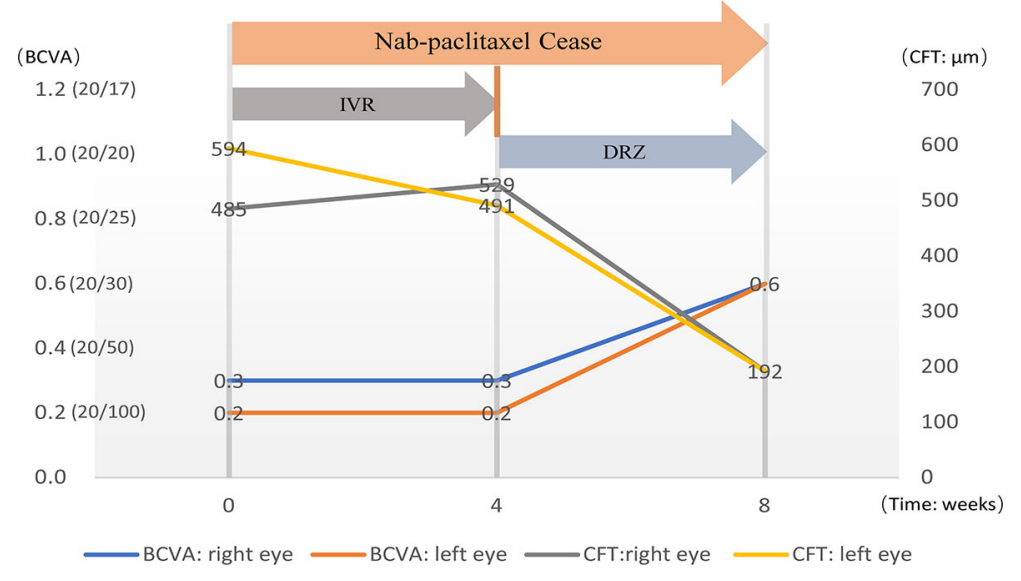

FIGURE 1 | The diagram shows the timeline of treatment and the changes in visual acuity and central retinal thickness. 

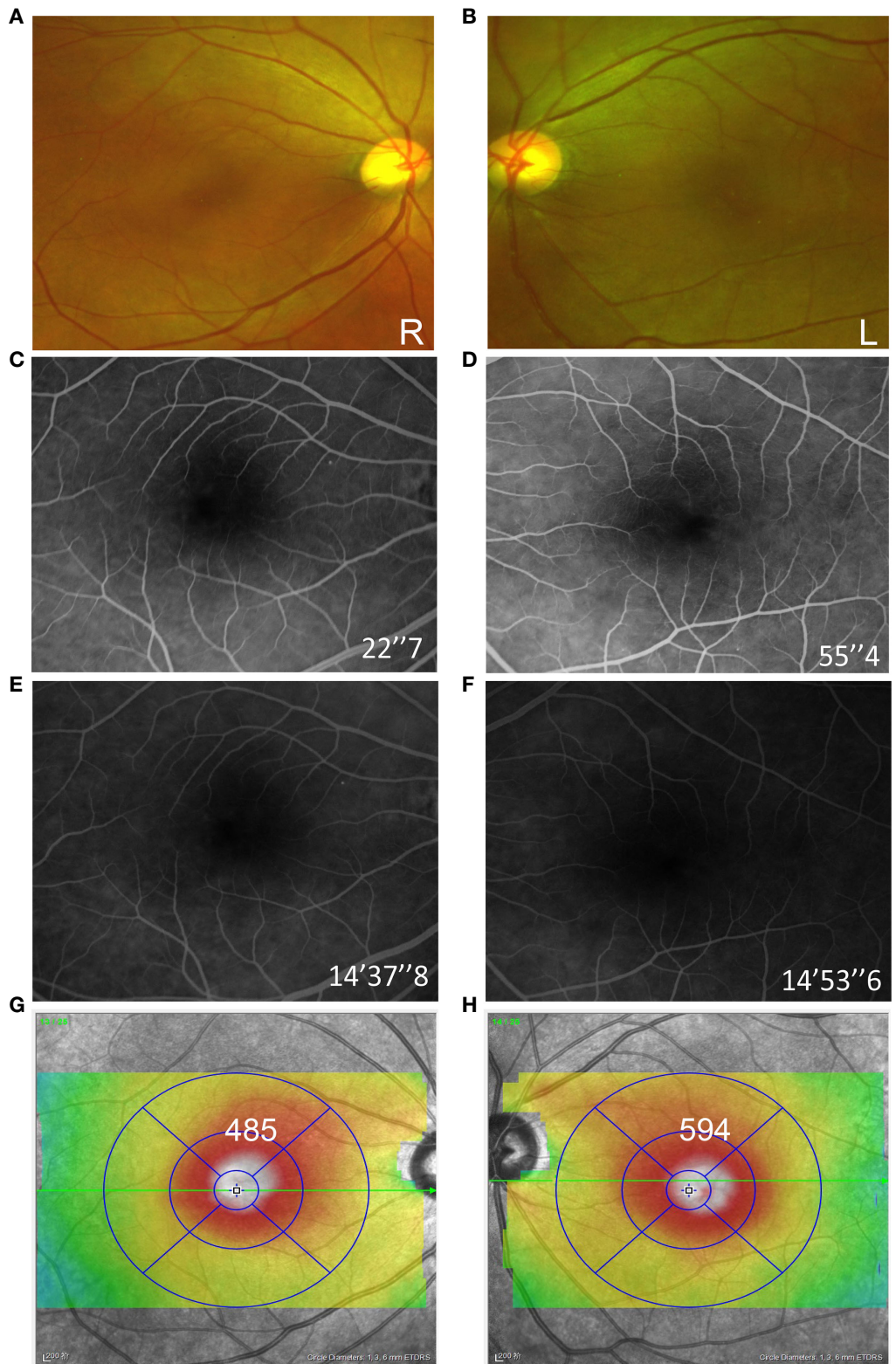

FIGURE 2 | Fundus examination of both eyes at the initial consultation. Ultra-wide field fundus image revealed macular edema in both eyes (A, B). The fluorescein angiograms did not reveal leakage from the parafoveal capillaries (C-F). Optical coherence tomography (OCT) B-scans showed the cystoid edema with a foveal thickness of $485 \mu \mathrm{m}$ on the right and $596 \mu \mathrm{m}$ on the left (G, H).

cases was from 3 weeks to 11 months, with a mean period of 2.8 months. In the cases without other treatments besides the cessation of nab-paclitaxel, the macular edema subsided within 1.5-6 months (2.6 months on average, $n=10)$, while the cases treated with DRZ seemed to experience a shorter recovery duration of 1-2 months (1.65 months on average, $n=6$ ). Moreover, the cases treated with oral glucose corticoid or intravitreal dexamethasone seemed to experience a longer resolution duration ( 0.75 to 11 months, $n=3$ ).

\section{DISCUSSION}

Taxanes, including docetaxel, cabazitaxel, paclitaxel, and nabpaclitaxel, are microtubule-stabilizing agents that are clinically effective against various malignancies (25). Paclitaxel was the first member of the taxoid family and was approved for use in chemotherapy in 1993 (26). Due to its hydrophobic nature, paclitaxel is poorly soluble. Its first licensed formulation used polymethylated castor oil (Cremophor EL, CrEL) and ethanol 


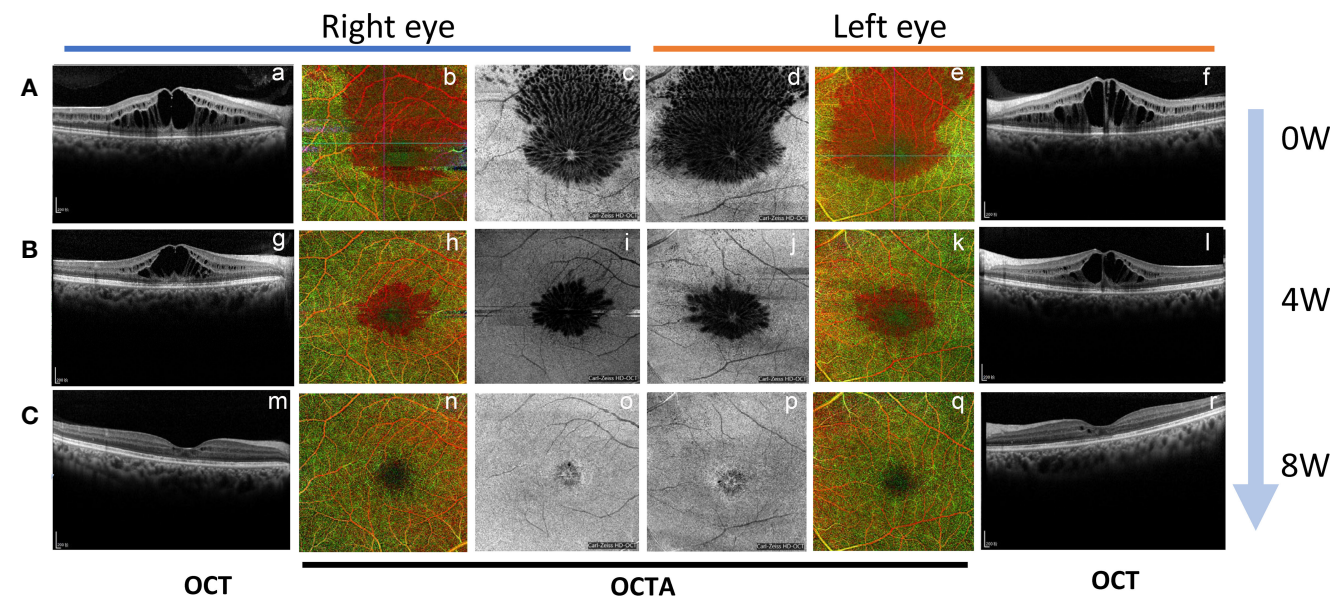

FIGURE 3 | The change of macular edema by B-scan and en face optical coherence tomography and OCTA during the time course. (A) The OCT B-scan (a, f), OCTA (b, e), and en face OCT (c, d) revealed macular edema in both eyes at initial consultation; (B) One month after applying IVR (OCT B-scan g and I, OCTA h and k, en face OCT i and j); (C) One month after topical brinzolamide in both eyes. (OCT B-scan $\mathrm{m}$ and r, OCTA $n$ and q, en face OCT o and p).

paclitaxel emulsification. However, CrEL can form tiny particles encapsulating paclitaxel molecules, affecting the absorption and utilization of tumor tissues. In addition, the degradation of CrEL may cause hypersensitivity reactions, requiring prolonged infusion times and premedication with steroids and antihistamines (27). Nab-paclitaxel is a newer formulation that uses albumin-bound nanoparticles to enhance paclitaxel solubility (2). A phase III study suggested that nab-paclitaxel has shown better efficacy in

TABLE 1 | Case reports of cystoid macular edema due to albumin-bound paclitaxel.

\begin{tabular}{|c|c|c|c|c|c|c|c|c|c|}
\hline No. & Author & Sex & Age(years) & Affected eye & Primary tumor & $\begin{array}{l}\text { Onset latency } \\
\text { (months) }\end{array}$ & $\begin{array}{c}\text { Nab-paclitaxel } \\
\text { Case }\end{array}$ & Other treatments & $\begin{array}{l}\text { Treatment time to } \\
\text { recovery (months) }\end{array}$ \\
\hline 1 & Risard et al. (5) & Male & 58 & Bilateral & Melanoma & 4 & Yes & $\mathrm{NO}$ & 1.5 \\
\hline 2 & Haider et al. (6) & Male & 73 & Bilateral & Lung & 42 & Yes & NO & 2 \\
\hline 3 & Fenicia et al. (7) & Female & 40 & Bilateral & Breast & 4 & - & DEX and DRZ & - \\
\hline 4 & Rahman et al. (8) & Female & 73 & Bilateral & Breast & 3 & No & IVB $^{\mathrm{a}}$ & Lost visit \\
\hline 5 & Smith et al. (9) & Female & 56 & Bilateral & Breast & 27 & Yes & NO & 3 \\
\hline 6 & Murphy et al. (10) & Female & 65 & Bilateral & Breast & 1.5 & Yes & NSAID and GC & 0.75 \\
\hline 7 & Murphy et al. (10) & Female & 58 & Bilateral & Breast & 11 & Yes & NO & 3 \\
\hline 8 & Rahimy and Sarraf (11) & Female & 32 & Bilateral & Breast & 9 & Yes & $\mathrm{NO}$ & 1.5 \\
\hline 9 & Park et al. (12) & Female & 69 & Bilateral & Breast & 6 & Yes & NO & 2 \\
\hline 10 & Rao and Choudhry (13) & Female & 45 & Bilateral & Breast & - & Yes & NO & Death \\
\hline 11 & Baskin and Garg (14) & Female & 40 & Bilateral & Breast & 6 & Yes & NSAID and GC & 4 \\
\hline 12 & Tanaka et al. (15) & Female & 47 & Bilateral & Breast & 4 & Yes & NO & 2 \\
\hline 13 & Matsuoka et al. (16) & Female & 39 & Bilateral & Breast & 8 & Yes & STTA & 11 \\
\hline 14 & Ehlers et al. (17) & Female & 59 & Bilateral & Breast & - & Yes & $\mathrm{DRZ}$ & 1 \\
\hline 15 & Hassall and Andrew (18) & Male & 73 & Bilateral & Hypopharynx & 2 & Yes & $\mathrm{DRZ}$ and $\mathrm{IVB}^{\mathrm{b}}$ & 2 \\
\hline 16 & Sridhar et al. (19) & Female & 48 & Bilateral & Pancreas & - & No & NO & - \\
\hline 17 & Lee et al. (20) & Female & 43 & Bilateral & Pancreas & 4 & Yes & NO & 3 \\
\hline 18 & Ota et al. (21) & Male & 71 & Bilateral & Pancreas & - & Yes & NO & 2 \\
\hline 19 & Ito et al. (22) & Female & 73 & Left Eye & Pancreas & 4 & Yes & NO & 6 \\
\hline 20 & Burgos-Blasco et al. (23) & Male & 67 & Bilateral & Pancreas & 6 & Yes & DEX & 4 \\
\hline 21 & Otsubo et al. (24) & Female & 72 & Bilateral & Breast & 2 & Yes & $\mathrm{DRZ}$ & $1.5^{\mathrm{c}}$ \\
\hline 22 & Otsubo et al. (24) & Male & 70 & Bilateral & Pancreas & 5 & Yes & DRZ & 1.25 \\
\hline 23 & Present case & Female & 45 & Bilateral & Breast & 18 & Yes & $\mathrm{DRZ}$ and IVR & 2 \\
\hline
\end{tabular}

NSAID, topical nonsteroidal anti-inflammatory drug; DEX, intravitreal dexamethasone; DRZ, topical dorzolamide; IVB, intravitreal bevacizumab; GC, topical corticosteroids; STTA, subtenon triamcinolone acetonide injection; IVR, intravitreal ranibizumab.

aIVB applied every 4 weeks: two injections for the right eye and three for the left.

${ }^{b} D R Z$ for the right eye and IVB monthly for the left eye.

${ }^{c} A$ total of 1.5 months for the right eye and 2.5 months for the left eye. 
antitumor with fewer adverse effects when compared to paclitaxel (28). Thus, albumin-bound paclitaxel is widely used in the chemotherapy of malignant tumors.

$\mathrm{T}$-CME is a rare side effect of taxanes, with an incidence of about $0.2-0.5 \%(3,4)$. The first case of T-CME was reported in 2003, caused by docetaxel (29). Then, CME caused by paclitaxel and nab-paclitaxel was first reported in 2007 (30) and 2008 (9), respectively. Nab-paclitaxel-related T-CME is mostly bilateral, with onset at 9.3 months (range: from 1.5 to 42 months) after applying albumin-bound paclitaxel.

It has been reported that, in $97.83 \%$ of the cases, no leakage was detected in fundus fluorescence angiography (FFA) or significant ICGA changes $(20,31)$. In all T-CME cases listed here (literature review and case report), which resulted from albumin-bound paclitaxel, no or minimal leakage was identified by FFA. As shown in the structural OCT B-scan, the hyporeflective cysts were located in the outer nuclear layer and inner nuclear layer, with intact contiguous outer plexiform layer and outer plexiform layer and retinal pigmented epithelium. Unlike CME caused by retinal vein occlusion or diabetic retinopathy, hard exudation in fundus photographs and hyperreflective plaques in OCT were not seen in T-CME cases, coinciding with the previous study (32). Taken together, increased capillary permeability may not be the primary cause of T-CME. Furthermore, though limited cases underwent OCTA examination, intact parafoveal capillary networks were detected without blood flow interruption. It indicated that TCME might not be a result of vascular degeneration of vessel occlusion.

Another hypothesis is that T-CME may associate with fluid retention syndrome, which is frequently seen in doxorubicin therapy and involves peripheral edema, weight gain, and fluid accumulation in the third space (pericardium, pleura, and ascites) (33). However, neither the previous nor our case was mentioned with combined thoracoabdominal fluid, and the present findings are insufficient to support this hypothesis.

Concerning treatment, the literature reviewed showed that some T-CME cases spontaneously resolved after discontinuing nab-paclitaxel without eye treatment within 1.5 weeks to 6 months $(5,6,9-13,19-21)$. Though the mechanism of inflammatory factors is uncertain, anti-inflammatory agents (steroids or NSAIDs) were applied previously. Nevertheless, those drugs were reported to be ineffective, and the patients even had a longer recovery time of 4 to 11 months (4 months on average), suggesting that inflammatory factors are not the primary cause of T-CME.

Regarding the anti-VEGF treatment, only two cases were reported with $\operatorname{IVB}(8,18)$ and the present case with IVR. In an interesting study conducted by Hassals et al., the patient received DRZ for the right eye and IVB monthly for the left eye, and CME subsided at around 2 months in both eyes (18). In our case, after being treated with ranibizumab, the area of the edema was narrowed. These data suggested that anti-VEGF may affect TCME but not critically.

Interestingly, six cases $(7,17,18,24)$, including the present case, treated with a combination of topical carbonic anhydrase inhibitor (CAI), seemed to experience a shorter recovery duration (1 to 2 months). In particular, Ehlers et al. reported a monocular control trial showing that CME in the eye treated with topical CAI resolved more rapidly than the other eye without extra medication after nab-paclitaxel cessation (17). In our case, when anti-VEGF was discontinued and switched to topical CAI, the macular edema was still getting better. These cases suggested that topical CAI may also have a therapeutic effect in nab-paclitaxel-induced CME. Although we cannot confirm whether the recovery was achieved by withdrawing the drug or the efficacy of the combined treatment with ranibizumab or CAI through a retrospective case report, we could still insist that CAI may have a therapeutic effect on T-CME. Nevertheless, we should also be aware that neither anti-VEGF nor CAI plays a crucial role in treating T-CME.

The underlying pathogenesis of T-CME remains unclear. Based on a careful analysis of published literature and cases, we propose that T-CME may be attributed to the disturbed distribution of the water channels in retinal Müller cells and retinal pigment epithelium (RPE), which is caused by the inhibitory effect of paclitaxel on microtubules and ultimately leads to retinal water transport impairment. Müller cells are responsible for the osmotic gradient in the neurosensory retina surrounding the intermediate capillary plexus and deep capillary plexus vessels. These cells possess a large number of water channels (34). Since the arrangement of these channels in cells depends on microtubule function, which is altered by paclitaxel, eventual edema occurs due to impaired water transport. This hypothesis may explain the possibility of recovery with nab-paclitaxel suspension. On the other hand, carbonic anhydrase (CA) XIV, an extracellular membranebound CA, was identified in retinal Müller cells, astrocytes, and the apical and basolateral membranes of the $\operatorname{RPE}(35,36)$. The author noted that CA XIV plays a role in $\mathrm{pH}$ and volume homeostasis in the extracellular space, and they suggested that CA XIV is the CAI target that enhances subretinal fluid absorption in macular edema (35). This is probably the reason why the recovery time looks shorter when combined with CAI treatment.

It is worth noting that there are also reports of no vision recovery even after macular edema has subsided after paclitaxel discontinuation (37). Moreover, in some cases of delayed CME diagnosis, macular degeneration progresses, and vision loss is irreversible (38).

\section{CONCLUSION}

Nab-paclitaxel is a commonly used anti-cancer drug, which may cause macular edema. Hence, patients should undergo eye examinations routinely, including visual acuity examinations and OCT or OCTA. FFA should be applied when necessary. For oncologists, it is important to be aware of the possibility of causing CME when using nab-paclitaxel. Furthermore, attention 
should be paid to differentiate from CME associated with paraneoplastic syndrome. Most T-CME would recover after suspending the drug; however, long-existing CME may result in irreversible vision loss with macular degeneration, and a combination of CAI or anti-VEGF may be considered for those long-existing CME.

\section{DATA AVAILABILITY STATEMENT}

The original contributions presented in the study are included in the article/supplementary material. Further inquiries can be directed to the corresponding authors.

\section{REFERENCES}

1. Li J, Mutanda I, Wang K, Yang L, Wang J, Wang Y. Chloroplastic Metabolic Engineering Coupled With Isoprenoid Pool Enhancement for Committed Taxanes Biosynthesis in Nicotiana Benthamiana. Nat Commun (2019) 10 (1):4850. doi: 10.1038/s41467-019-12879-y

2. Cecco S, Aliberti M, Baldo P, Giacomin E, Leone R. Safety and Efficacy Evaluation of Albumin-Bound Paclitaxel. Expert Opin Drug Saf (2014) 13 (4):511-20. doi: 10.1517/14740338.2014.893293

3. Fortes BH, Liou H, Dalvin LA. Ophthalmic Adverse Effects of Taxanes: The Mayo Clinic Experience. Eur J Ophthalmol (2020) 1120672120969045. doi: 10.1177/1120672120969045

4. Kaya M, Atas F, Gulsum Guc Z, Oztop I, Durak I, Saatci AO. A CrossSectional Optical Coherence Tomography Study in Patients on Taxane-Based Therapy and a Case Report With the Literature Review. Cutan Ocul Toxicol (2020) 39(3):287-93. doi: 10.1080/15569527.2020.1790592

5. Risard SM, Pieramici DJ, Rabena MD. Cystoid Macular Edema Secondary to Paclitaxel (Abraxane). Retin Cases Brief Rep (2009) 3(4):383-5. doi: 10.1097/ ICB.0b013e318193492c

6. Haider A, Bababeygy SR, Lu SY. Cystoid Macular Edema and Macular Pigmentation Associated With Nab-Paclitaxel Therapy. Retin Cases Brief Rep (2015) 9(3):220-2. doi: 10.1097/icb.0000000000000143

7. Fenicia V, Verrilli S, Recupero SM. Nab-Paclitaxel Induced Cystoid Macular Edema: Clinical Experience With Intravitreal Implant of Dexamethasone vs Topical Dorzolamide. Ophthalmologica (2014) 232:49-50.

8. Rahman HT, Yeh S, Bergstrom CS. Cystoid Macular Edema Without Leakage Secondary to Nab-Paclitaxel (Abraxane): Clinical Experience With Intravitreal Bevacizumab. J Ocul Pharmacol Ther (2013) 29(3):360-2. doi: 10.1089/jop.2011.0178

9. Smith SV, Benz MS, Brown DM. Cystoid Macular Edema Secondary to Albumin-Bound Paclitaxel Therapy. Arch Ophthalmol (Chicago Ill 1960) (2008) 126(11):1605-6. doi: 10.1001/archopht.126.11.1605

10. Murphy CG, Walsh JB, Hudis CA, Lake D, Theodoulou M. Cystoid Macular Edema Secondary to Nab-Paclitaxel Therapy. J Clin Oncol (2010) 28(33): e684-7. doi: 10.1200/jco.2010.30.3750

11. Rahimy E, Sarraf D. Cystoid Macular Edema Secondary to Nanoparticle Albumin-Bound Paclitaxel Therapy. Ophthalmic Surg Lasers Imaging Retina (2013) 44(2):187-9. doi: 10.3928/23258160-20130212-02

12. Park E, Goldberg NR, Adams S. Nab-Paclitaxel-Induced Cystoid Macular Edema in a Patient With Pre-Existing Optic Neuropathy. Anticancer Drugs (2016) 27(6):580-4. doi: 10.1097/cad.0000000000000361

13. Rao RC, Choudhry N. Cystoid Macular Edema Associated With Chemotherapy. CMAJ (2016) 188(3):216. doi: 10.1503/cmaj.131080

14. Baskin DE, Garg SJ. Abraxane-Induced Cystoid Macular Edema Refractory to Concomitant Intravenous Bevacizumab. Can J Ophthalmol (2011) 46(2):2001. doi: 10.3129/i11-001

\section{ETHICS STATEMENT}

Written informed consent was obtained from the individual(s) for the publication of any potentially identifiable images or data included in this article.

\section{AUTHOR CONTRIBUTIONS}

SY and QF drafted the manuscript. JY and JX contributed to the acquisition of the data and clinical assessment. ST and JHM analyzed the review data and critically revised the manuscript. All authors contributed to the article and approved the submitted version.

15. Tanaka Y, Bando H, Hara H, Ito Y, Okamoto Y. Cystoid Macular Edema Induced by Nab-Paclitaxel. Breast Cancer (Tokyo Japan) (2015) 22(3):324-6. doi: 10.1007/s12282-012-0373-y

16. Matsuoka N, Hasebe H, Mayama T, Fukuchi T. Sub-Tenon Injections of Triamcinolone Acetonide Had Limited Effect on Cystoid Macular Edema Secondary to Nanoparticle Albumin-Bound-Paclitaxel (Abraxane). Case Rep Ophthalmol Med (2015) 2015:181269. doi: $10.1155 / 2015 / 181269$

17. Ehlers JP, Rayess H, Steinle N. Topical Dorzolamide Therapy for TaxaneRelated Macular Oedema. Eye (London England) (2013) 27(1):102-4 doi: 10.1038/eye.2012.228

18. Hassall MM, Andrew NH. Single-Eye Trial of a Topical Carbonic Anhydrase Inhibitor Versus Intravitreal Bevacizumab for the Treatment of Taxane DrugInduced Cystoid Macula Oedema. BMJ Case Rep (2016) 2016:10.1136/bcr2015-212733. doi: 10.1136/bcr-2015-212733

19. Sridhar J, Shahlaee A, Ehmann D, Samara WA, Rahimy E, Ho AC, et al. En Face Optical Coherence Tomography and Optical Coherence Tomography Angiography Imaging of Taxane-Associated Cystoid Macular Edema. Ophthalmic Surg Lasers Imaging Retina (2016) 47(2):176-9. doi: 10.3928/ 23258160-20160126-12

20. Lee J, Ra H, Baek J. Ultra-Widefield Angiographic Imaging of AlbuminBound Paclitaxel-Induced Cystoid Macular Edema. Indian J Ophthalmol (2019) 67(12):2058-9. doi: 10.4103/ijo.IJO_734_19

21. Ota S, Tanke G, Ito R, Hara K, Takada Y, Adachi K, et al. Cystoid Macular Edema Secondary to Albumin-Bound Paclitaxel Therapy for Pancreatic Cancer. Nihon Shokakibyo Gakkai Zasshi (2021) 118(3):272-8. doi: 10.11405/nisshoshi.118.272

22. Ito T, Deguchi K, Yoshii K, Kashii M. A Case of Cystoid Macular Edema Secondary to Albumin-Bound Paclitaxel Therapy. Gan To Kagaku Ryoho (2017) 44(7):599-602.

23. Burgos-Blasco B, Hernandez-Ruiz S, Lopez-Guajardo L, Donate-Lopez J. Dexamethasone Intravitreal Implant in Cystoid Macular Edema Secondary to Paclitaxel Therapy. Am J Ophthalmol Case Rep (2020) 18:100653. doi: 10.1016/j.ajoc.2020.100653

24. Otsubo M, Kinouchi R, Kamiya T, Yoshida A. Regression of TaxaneRelated Cystoid Macular Edema After Topical Dorzolamide Treatment: Two Case Reports. J Med Case Rep (2021) 15(1):355. doi: 10.1186/s13256021-02954-8

25. Rzeszotarska A, Stodolska-Nowak A, Kufel-Grabowska J, Nowakowski B, Kocięcki J. Ocular Side Effects of the Taxane-Based Chemotherapy - do Only Vascular Disorders Matter? OncoReview (2019) 9(3):59-63. doi: 10.24292/ 01.Or.319200619

26. Rowinsky EK, Donehower RC. Paclitaxel (Taxol). N Engl J Med (1995) 332 (15):1004-14. doi: 10.1056/NEJM199504133321507

27. Gelmon K. The Taxoids: Paclitaxel and Docetaxel. Lancet (1994) 344 (8932):1267-72. doi: 10.1016/S0140-6736(94)90754-4

28. Gradishar WJ, Tjulandin S, Davidson N, Shaw H, Desai N, Bhar P, et al. Phase III Trial of Nanoparticle Albumin-Bound Paclitaxel Compared With 
Polyethylated Castor Oil-Based Paclitaxel in Women With Breast Cancer. J Clin Oncol (2005) 23(31):7794-803. doi: 10.1200/JCO.2005.04.937

29. Teitelbaum BA, Tresley DJ. Cystic Maculopathy With Normal Capillary Permeability Secondary to Docetaxel. Optom Vis Sci (2003) 80(4):277-9. doi: 10.1097/00006324-200304000-00004

30. Joshi MM, Garretson BR. Paclitaxel Maculopathy. Arch Ophthalmol (Chicago Ill 1960) (2007) 125(5):709-10. doi: 10.1001/archopht.125.5.709

31. Álvarez-Fernández D, Cubillas-Martín M, Álvarez-Suárez ML, ViescasFernández MJ, Medina-Mejías MR, Rodríguez-Balsera C. Macular Oedema Associated With Taxanes: A Case Report and Literature Review. Arch Soc Esp Oftalmol (2020) 95(10):485-95. doi: 10.1016/j.oftal. 2020.05.030

32. Perez JM, Teo K, Ong R, Maruyama-Inoue M, Freund KB, Tan ACS. Optical Coherence Tomography Characteristics of Taxane-Induced Macular Edema and Other Multimodal Imaging Findings. Graefes Arch Clin Exp Ophthalmol (2020) 258(8):1607-15. doi: 10.1007/s00417-020-04755-Z

33. Semb KA, Aamdal S, Oian P. Capillary Protein Leak Syndrome Appears to Explain Fluid Retention in Cancer Patients Who Receive Docetaxel Treatment. J Clin Oncol (1998) 16(10):3426-32. doi: 10.1200/JCO.1998. 16.10 .3426

34. Goodyear MJ, Crewther SG, Junghans BM. A Role for Aquaporin-4 in Fluid Regulation in the Inner Retina. Vis Neurosci (2009) 26(2):159-65. doi: 10.1017/S0952523809090038

35. Nagelhus EA, Mathiisen TM, Bateman AC, Haug FM, Ottersen OP, Grubb $\mathrm{JH}$, et al. Carbonic Anhydrase XIV Is Enriched in Specific Membrane Domains of Retinal Pigment Epithelium, Muller Cells, and Astrocytes. Proc Natl Acad Sci U S A (2005) 102(22):8030-5. doi: 10.1073/pnas. 0503021102
36. Ochrietor JD, Clamp MF, Moroz TP, Grubb JH, Shah GN, Waheed A, et al. Carbonic Anhydrase XIV Identified as the Membrane CA in Mouse Retina: Strong Expression in Müller Cells and the RPE. Exp Eye Res (2005) 81(4):492500. doi: 10.1016/j.exer.2005.03.010

37. Georgakopoulos CD, Makri OE, Vasilakis P, Exarchou A. Angiographically Silent Cystoid Macular Oedema Secondary to Paclitaxel Therapy. Clin Exp Optom (2012) 95(2):233-6. doi: 10.1111/j.1444-0938.2011.00672.x

38. Yokoe T, Fukada I, Kobayashi K, Shibayama T, Miyagi Y, Yoshida A, et al. Cystoid Macular Edema During Treatment With Paclitaxel and Bevacizumab in a Patient With Metastatic Breast Cancer: A Case Report and Literature Review. Case Rep Oncol (2017) 10(2):605-12. doi: 10.1159/000477897

Conflict of Interest: The authors declare that the research was conducted in the absence of any commercial or financial relationships that could be construed as a potential conflict of interest.

Publisher's Note: All claims expressed in this article are solely those of the authors and do not necessarily represent those of their affiliated organizations, or those of the publisher, the editors and the reviewers. Any product that may be evaluated in this article, or claim that may be made by its manufacturer, is not guaranteed or endorsed by the publisher.

Copyright $\odot 2021$ Ye, Fang, Yao, Xing, Tang and Ma. This is an open-access article distributed under the terms of the Creative Commons Attribution License (CC BY). The use, distribution or reproduction in other forums is permitted, provided the original author(s) and the copyright owner(s) are credited and that the original publication in this journal is cited, in accordance with accepted academic practice. No use, distribution or reproduction is permitted which does not comply with these terms. 